\title{
Structural Study of Surfactant-dependent Interaction with Protein
}

\author{
Sumit Mehan ${ }^{1}$, Vinod K. Aswal ${ }^{1, *}$ and Joachim Kohlbrecher ${ }^{2}$ \\ ${ }^{1}$ Solid State Physics Division, Bhabha Atomic Research Centre, Mumbai 400 085, India \\ ${ }^{2}$ Laboratory for Neutron Scattering, Paul Scherrer Institut, CH-5232 PSI Villigen, Switzerland \\ "E-mail:vkaswal@barc.gov.in
}

\begin{abstract}
Small-angle neutron scattering (SANS) has been used to study the complex structure of anionic BSA protein with three different (cationic DTAB, anionic SDS and non-ionic C12E10) surfactants. These systems form very different surfactant-dependent complexes. We show that the structure of protein-surfactant complex is initiated by the site-specific electrostatic interaction between the components, followed by the hydrophobic interaction at high surfactant concentrations. It is also found that hydrophobic interaction is preferred over the electrostatic interaction in deciding the resultant structure of protein-surfactant complexes.
\end{abstract}

Keywords: Protein solutions, Self assembly, protein-surfactant complexes, SANS

PACS: 87.15.hp, 81.16.Fg, 61.05.fg

\section{INTRODUCTION}

The interaction of surfactants with protein is usually known to unfold the protein with formation of protein-surfactant complexes [1]. These complexes find their usefulness in many fields including pharmaceuticals, cosmetics and protein separation. There are numerous studies of ionic surfactants with proteins to study different aspects of protein-surfactant complexes under different solution conditions. Nonionic surfactants show very weak or no interactions with proteins [2]. The nature of binding of surfactant on protein is governed by electrostatic and hydrophobic interactions. The properties of these complexes can be tuned by varying these interactions. However, it is not clear how these two interactions (electrostatic and hydrophobic) compete in forming the structure of protein-surfactant complexes. Proteins are non-uniformly charged molecules (comprising both positive and negative patches) and take a folded three dimensional conformation (hydrophobic groups hidden in the core) in native conditions. Therefore, the charge nature of surfactant and its hydrophobicity could be important in deciding the protein-surfactant interaction and their resultant structure. Herein, we have studied the structure and interaction of protein-surfactant complexes for anionic BSA protein with three different surfactants (cationic DTAB, anionic SDS and non-ionic C12E10) by small-angle neutron scattering (SANS). The three surfactants used have the same hydrophobicity $\left(\mathrm{C}_{12} \mathrm{H}_{25}\right.$ alkyl chain), but differ in the charge nature of the head group. SANS is a powerful technique to study different length scales in such hydrogenous systems in the range 1-100 $\mathrm{nm}[3]$.

\section{EXPERIMENTAL}

Bovine serum albumin (BSA) protein and surfactants [cationic dodecyl trimethyl ammonium bromide (DTAB), anionic sodium dodecyl sulphate (SDS) and non-ionic decaethylene glycol monododecyl ether (C12E10)] were purchased from Sigma-Aldrich. The samples were prepared by dissolving weighted amount of protein $(1 \mathrm{wt} \%)$ and surfactants $(40 \mathrm{mM})$ at $0.2 \quad \mathrm{M} \quad \mathrm{NaCl}$ and $20 \mathrm{mM}$ phosphate buffer $(\mathrm{pH}=7)$ in $\mathrm{D}_{2} \mathrm{O}$. The presence of salt reduces the electrostatic interaction between the components. The use of $\mathrm{D}_{2} \mathrm{O}$ over $\mathrm{H}_{2} \mathrm{O}$ provides low incoherent background and better contrast for hydrogenous samples. Small-angle neutron scattering experiments were performed at the SANS-I facility, Swiss Spallation Neutron Source SINQ, Paul Scherrer Institut, Switzerland [4]. The wavelength $(\lambda)$ of neutron beam used was $8 \AA$ and the scattered neutrons from samples were detected using two-dimensional $96 \mathrm{~cm} \times 96 \mathrm{~cm}$ detector. Data were collected at two sample-to-detector distances of 2 and $8 \mathrm{~m}$ to cover a wave vector transfer range of $0.005-0.3 \AA^{-1}$. Corrections were made for background and empty cell contributions and data were normalized to absolute cross-sectional unit using standard procedure. 


\section{SANS ANALYSIS}

In SANS experiments, one measures the differential scattering cross-section per unit volume $d \Sigma / d \Omega$ as a function of $Q$. For a system of monodisperse particles in a solvent, $d \Sigma / d \Omega$ can be expressed as

$$
\frac{d \Sigma}{d \Omega}(Q)=\phi V_{p} \Delta \rho^{2} P(Q) S(Q)+B
$$

where $\varnothing$ is volume fraction of particles, $V_{p}$ is particle volume and $\Delta \rho^{2}$ is scattering contrast of particles. $P(Q)$ and $S(Q)$ are the form factor and structure factor, respectively. $B$ is the incoherent background, which predominately arises due to presence of hydrogen in the sample.

In the case of ellipsoidal particles with its semi axes $R$ and $\varepsilon R, P_{e}(Q)$ can be written as

$$
\begin{gathered}
P_{e}(Q)=\int_{0}^{\pi / 2} P_{s}(Q, r) \sin \beta d \beta \\
r=R \sqrt{\sin ^{2} \beta+\varepsilon^{2} \cos ^{2} \beta}
\end{gathered}
$$

where $\varepsilon<1$ for oblate ellipsoidal and $\varepsilon>1$ for prolate ellipsoidal and $\varepsilon=1$ for spherical shape of particles. $\beta$ is the angle between semi-major axis and wave vector transfer.

The $S(Q)$ for mass fractal structure is given by

$$
S_{f}(Q)=1+\frac{1}{\left(Q r_{0}\right)^{D}} \frac{D \Gamma(D-1)}{\left[1+\frac{1}{(Q \xi)^{2}}\right]^{(D-1) / 2}} \sin \left\{(D-1) \times \tan ^{-1}(Q \xi)\right\}
$$

where $D$ is fractal dimension, $\xi$ represents the correlation length and $r_{0}$ is characteristic dimension of individual particles. $\Gamma$ represents the mathematical gamma function.

The modeled scattering profiles were smeared by the appropriate resolution function to compare with the experimental data. The parameters in the analysis were optimized by means of nonlinear least-square fitting program.

\section{RESULTS AND DISCUSSION}

Figure 1 shows the SANS data of pure components of BSA protein and three surfactants (cationic DTAB, anionic SDS, non-ionic C12E10) in $\mathrm{D}_{2} \mathrm{O}$. The different $Q$ dependence of the data suggests the different structure and interaction of these systems. The BSA protein is fitted with the form factor $P(Q)$ of oblate ellipsoidal particles with semi-major axis and semi-minor axis as $42.3 \AA$ and $13.6 \AA$, respectively.
The C12E10 micelles are fitted with spherical core shell structure with core radius and shell thickness as $17.3 \AA$ Á and $12.2 \AA$, respectively. The ionic surfactant micelles, DTAB and SDS are fitted with prolate ellipsoidal core-shell structure with semi-minor axis of $16.7 \AA$ for both, semi-major axes of $21.0 \AA$ A, $28.3 \AA$ and shell thickness of $5.8 \AA \AA$, $5.0 \AA \AA$, respectively. The data of ionic micelles show a contribution of $S(Q)$, which has been fitted using screen Coulomb interaction potential [3]. The effective charge on DTAB and SDS micelles is found to be 21 e.u. and 25 e.u., respectively. The calculated aggregation numbers of DTAB, SDS and C12E10 micelles are 70, 94, 60, respectively. Proteins and surfactants share the common property of both having charged groups (or hydrophilic groups) and hydrophobic portions. The present combination of protein and surfactants are treated as model system because of comparable electrostatic or hydrophobic interactions in these systems [5].

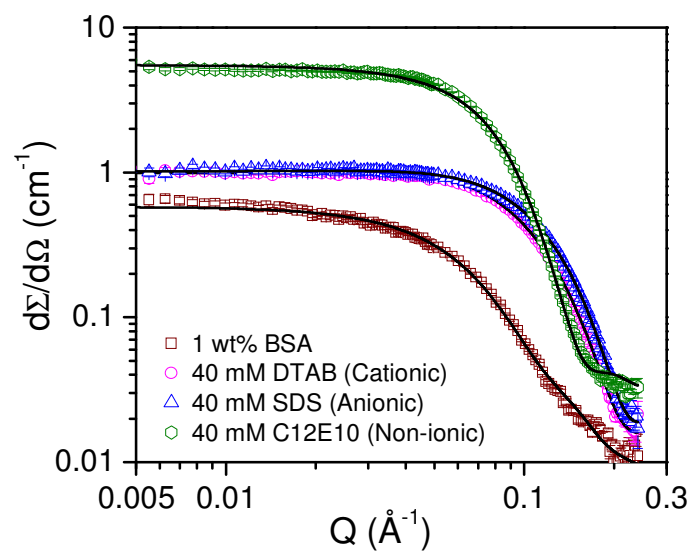

FIGURE 1. SANS data of 1 wt $\%$ BSA protein and $40 \mathrm{mM}$ surfactants (cationic DTAB, anionic SDS and non-ionic C12E10) in $\mathrm{D}_{2} \mathrm{O}$.

SANS data from the complexes of $1 \mathrm{wt} \% \mathrm{BSA}$ protein with $40 \mathrm{mM}$ of cationic DTAB, anionic SDS and non-ionic C12E10 micelles is shown in Figure 2. These data clearly show that the interaction of BSA protein with three surfactants is different. In particular, the features of ionic surfactants (both cationic and anionic) with protein are very different from that of non-ionic surfactant with protein. The scattering profiles of BSA protein with ionic surfactants show linearity in intermediate $Q$ range. The linearity on log-log scale indicates the presence of fractal kind of structure. The fractal structure is representation of bead-necklace model of protein-surfactant complex, where micelle-like clusters of surfactant adsorbed along the unfolded protein chain in their interaction [6]. The high $Q$ and low $Q$ cut-off of linearity give the information about size of micelle-like clusters and overall size of complex (correlation length), 
respectively. The slope of linearity gives the information about packing of micelle-like clusters adsorbed along the unfolded protein chain. The shifting of low $Q$ cut-off to lower $Q$ values for the case of DTAB as compared to SDS indicates that the overall size of complex is increased and protein unfolding is enhanced in case of cationic DTAB as compared to anionic SDS in interaction with anionic BSA protein. The fitting parameters are shown in table 2. In contrast to the ionic surfactant, the scattering profile of non-ionic C12E10 surfactant does not show any region of linearity. The scattering profile of BSA-C12E10 complex is fitted with coexistence of C12E10 micelles with BSA protein in solution. The similar features of data of cationic and anionic surfactants with anionic protein and no interaction of non-ionic surfactant suggest the surfactant-protein interaction is electrostatic driven. It works for both cationic and anionic surfactants because of site specific nature of electrostatic interaction with protein. The fact that charge neutralization on protein in case of oppositely charged surfactant is expected to be higher, which results in higher interaction of DTAB with BSA protein.

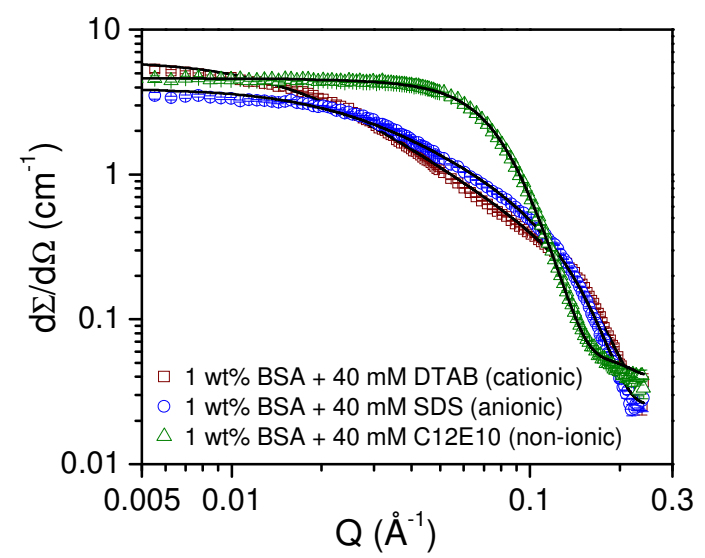

FIGURE 2. SANS data of protein-surfactant complexes of $1 \mathrm{wt} \%$ BSA protein with $40 \mathrm{mM}$ surfactants (cationic DTAB, anionic SDS and non-ionic C12E10) in $\mathrm{D}_{2} \mathrm{O}$.

TABLE 2. The structural parameters of protein-surfactant complexes of BSA protein with ionic surfactants.

\begin{tabular}{cccc}
\hline Sample & $\begin{array}{c}\text { Micelle } \\
\text { Radius } \\
r_{0}(\hat{A})\end{array}$ & $\begin{array}{c}\text { Fractal } \\
\text { Dimension } \\
D\end{array}$ & $\begin{array}{c}\text { Correlation } \\
\text { length } \\
\xi(\AA)\end{array}$ \\
\hline BSA-DTAB & 16.8 & 1.81 & 56.2 \\
BSA-SDS & 18.6 & 1.71 & 37.5 \\
\hline
\end{tabular}

It has been shown that the structure of proteinsurfactant complex (Figure 2) depends on electrostatic interaction (binding of surfactant to protein) and hydrophobic interaction (bead necklace structure of complex) of the components. To examine the role of these two interactions, Figure 3 shows SANS data of BSA protein with mixed non-ionic and ionic surfactant systems. It is observed that the interaction of ionic surfactant with protein is completely suppressed in presence of non-ionic surfactant. The data of mixed $(\mathrm{C} 12 \mathrm{E} 10+\mathrm{DTAB})$ and $(\mathrm{C} 12 \mathrm{E} 10+\mathrm{SDS})$ surfactant systems with BSA protein are similar to that of C12E10 with BSA protein. The differences of mixed systems with that of C12E10 are because of different structure of micelles in these systems (inset of Figure 3). In all these systems, individual micelles coexist with unperturbed protein. These results can be understood on the basis that hydrophobic interaction for ionic surfactant is preferred over the electrostatic interaction, which results for ionic surfactant to form individual mixed micelles with non-ionic surfactant than their any electrostatic interaction with protein.

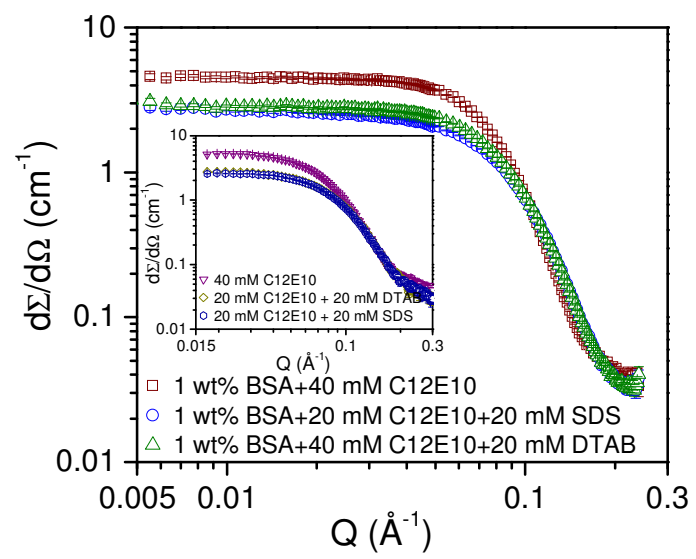

FIGURE 3. SANS data of protein-surfactant complexes of 1 wt $\%$ BSA protein with $40 \mathrm{mM}$ mixed surfactants (C12E10-DTAB and $\mathrm{C} 12 \mathrm{E} 10-\mathrm{SDS})$ in $\mathrm{D}_{2} \mathrm{O}$. Inset shows SANS data of mixed surfactants without BSA protein.

In summary, the interaction of surfactant with protein is initiated by electrostatic interactions and followed by hydrophobic interactions at higher surfactant concentration. In the absence of electrostatic interactions, non-ionic surfactant does not any interaction with protein. In the mixed micelles, ionic surfactants prefer hydrophobic interactions with nonionic micelles over electrostatic binding with protein.

\section{REFERENCES}

1. S. H. Chen and J. Teixeira, Phys. Rev. Lett. 57, 25832586 (1986).

2. A. D. Nielsen, K. Borch and P. Westh, Biochimica et Biophysica Acta 1479, 321-331 (2000).

3. S. Mehan, A. J. Chinchalikar, S. Kumar, V. K. Aswal and R. Schweins, Langmuir 29, 11290-11299 (2013).

4. J. Kohlbrecher and W. Wagner, J. Appl. Cryst. 33, 804806 (2000).

5. V.K. Aswal, S. Chodankar, V. Ronny, J. Kohlbrecher and A.G. Wagh, Phys. Rev. E 80, 011924 (2009).

6. S. Chodankar, V. K. Aswal, J. Kohlbrecher, R. Vavrin and A. G. Wagh, J. Phys.: Condens. Matter 19, 326102 (2007). 